\title{
EL PRETÉRITO PERFECTO COMPUESTO EN EL ÁMBITO \\ HISPÁNICO
}


La semántica del pretérito perfecto compuesto ha constituido tradicionalmente una de las cuestiones más abordadas en la gramática del español, debido principalmente a su alternancia con una forma simple: el pretérito indefinido. A pesar de todo, y posiblemente porque aún no se ha logrado un esquema descriptivo adecuado, siguen proliferando las obras en las que se introducen nuevas hipótesis o se perfeccionan y se reelaboran las ya existentes. No obstante, la mayoría de ellas no ofrecen un panorama general del ámbito hispánico ni consideran factores de importancia como son los procesos de gramaticalización.

Palabras Clave: pretérito perfecto compuesto, pretérito indefinido, gramaticalización, tiempo, aspecto

The analysis of the Spanish present perfect (pretérito perfecto compuesto) is one of the topics that has traditionally received the most attention among linguists, due to its differences with the past simple: the so-called pretérito indefinido. Even though, it seems that we are far from having found a satisfactory explanation, since a large number of studies on this subject tend to introduce new hypothesis or even to improve the existent ones. Unfortunately, the greater part of them do not take into account neither the whole Hispanic domain nor important factors such as grammaticalization processes.

KEY WORDS: present perfect, past simple, grammaticalization, tense, aspect

RECEPCIÓN: 11/01/2015

ACEPTACIÓN: 13/03/2015 


\title{
EL PRETÉRITO PERFECTO COMPUESTO EN EL ÁMBITO HISPÁNICO
}

\author{
Juan Moreno Burgos \\ Universität Regensburg / HAW Landshut
}

\section{Introducción ${ }^{1}$}

El pretérito perfecto compuesto (en adelante PPC) en el español estándar peninsular constituye una forma polisémica cuyas funciones se solapan parcialmente con las del pretérito indefinido ${ }^{2}$ (en adelante PI). Se trata de un caso que podríamos calificar de "invasión" semántica, dado que el valor original de la forma compuesta se corresponde con el aspecto Perfecto. ${ }^{3}$ Esta apreciación inicial no es sin embargo aplicable al español hablado en Latinoamérica, debido a que en la mayoría de los países de dicho continente es significativo el empleo del PI allí donde el español peninsular opta a

\footnotetext{
${ }^{1}$ Agradezco los comentarios de dos revisores anónimos, los cuales han contribuido a un mejora de la versión final de este trabajo.

${ }^{2}$ Por razones de claridad expositiva, emplearemos esta denominación, y no la de pretérito perfecto simple.

${ }^{3}$ Siguiendo la convención, nos valdremos de mayúsculas al aludir a las diferentes variedades aspectuales.
} 
menudo por el PPC (cf. Moreno de Alba, 2006: 18). Esto es, mientras que en Madrid se escuchan oraciones como Hoy he comido pasta o Juan ya ha llegado, en Guanajuato se prefiere decir Hoy comí pasta y Juan ya llegó, respectivamente.

El uso de la forma compuesta en Latinoamérica no está, sin embargo, completamente excluido, tal y como observamos en la siguiente oración:

(1) A raíz de la crisis financiera de 1994, han surgido nuevos sistemas de financiamiento de vivienda. La autoconstrucción es el modelo más recurrente, pero en los últimos dos años han surgido 31 empresas de autofinanciamiento [CREA: México]. ${ }^{4}$

El objetivo de este artículo será, por lo tanto, reflexionar por qué en casos como este, el hablante elige dicha forma verbal, dado que, si bien a primera vista no sería llamativo para un hablante madrileño, los datos referidos hasta ahora nos indican que aquí deben existir motivaciones semánticas diferentes. En este sentido, ya se han aventurado diferentes respuestas: por un lado, autores como Martínez-Atienza (2008) encuentran oportuno realizar un análisis semántico similar al del present perfect inglés, mediante el cual se considera que una situación pasada puede seguir siendo válida en el momento presente (Perfecto Continuativo). Por otro lado, autores como Rodríguez Louro (2009) opinan que es posible vincular al PPC con el ámbito de lo vivido por el

\footnotetext{
${ }^{4}$ Salvo que se indique lo contrario, tomaremos los ejemplos del corpus CREA de la Real Academia Española, indicando entre corchetes la procedencia geográfica de los mismos: España o México. Nos hemos decidido por este país latinoamericano avalados por los estudios de Lope Blanch $(1961,1992)$.
} 
sujeto de la predicación (Perfecto Experiencial). ${ }^{5}$ Nosotros explicaremos por qué ninguna de estas dos hipótesis deben ser trasladadas al ejemplo (1): ni podemos afirmar que el PPC posea la misma semántica que la correspondiente forma compuesta inglesa ni está claro que la citada lectura experiencial pueda ser accesible desde cualquiera de las variedades del español. Es cierto que la segunda de las autoras se centra en el español rioplatense, pero se hace necesario reflexionar sobre la inconveniencia de establecer una analogía con el español mexicano.

Nuestro plan de trabajo será el siguiente: tras presentar las teorías tempo-aspectuales de las que partimos, detallaremos la semántica del PPC en el ámbito peninsular. A continuación nos centraremos en el PPC en el continente americano (en concreto, México), con el fin de demostrar que se registran significados diferentes. Para ello indicaremos que esta forma aparece aquí relacionada con la expresión de diferentes fases, las cuales pueden hacerse explícitas mediante el uso de la perífrasis <estar + gerundio $>$. De modo paralelo, consideraremos que el PPC se emplea como un mecanismo para reducir el contenido de verdad de una proposición en el momento del habla. Finalmente, abordaremos el Perfecto Experiencial, para llegar a la conclusión de que se trata originalmente de un pretérito que recibe una reinterpretación aspecto-modal. Relacionado con ello están los enunciados

\footnotetext{
${ }^{5}$ El Continuativo y el Experiencial, junto al Resultativo, son las tres subvariedades que, según autores como Fenn (1987) y Comrie (1976), se atribuyen al aspecto Perfecto.
} 
que incluyen el adverbio siempre. De ello nos encargaremos en el último apartado.

\section{Aspecto y tiempo gramaticales}

\subsection{Generalidades}

En este artículo consideraremos, en contra de lo que opinan Rojo (1974) o Veiga (1992), que el aspecto ocupa un lugar importante en el sistema verbal del español. Se trata de una categoría gramatical que, a diferencia del tiempo, se caracteriza por poner en relieve diferentes fases del desarrollo temporal, independientemente de cuándo se produzca su anclaje (esto es, en el pasado, en el presente o en el futuro). Ambas categorías, la de tiempo y la de aspecto, deben ser contempladas como complementarias.

Los autores en los que nos apoyaremos para presentar estas teorías serán Klein (1992) y Reichenbach (1947), respectivamente. Según el primero de ellos, el aspecto se puede describir como una relación entre el Tiempo de la Situación y el Tiempo del Foco; esto es, entre un evento y la parte que se selecciona de él. Esto da lugar a las combinaciones siguientes: Imperfecto (se focaliza una parte interna del Tiempo de la Situación), Aoristo (el Tiempo del Foco coincide con el Tiempo de la Situación), Perfecto (el Tiempo del Foco sucede al Tiempo de la Situación) y Prospectivo (el Tiempo del Foco precede al Tiempo de la Situación). ${ }^{6}$ Desde las ideas

\footnotetext{
${ }^{6}$ Es conveniente, sin embargo, no perder de vista que en esta descripción el Tiempo de la Situación se corresponde más bien con los eventos (situaciones di-
} 
reichenbachianas, el tiempo gramatical debe ser considerado como una categoría anafórico-deíctica que surge como relación entre tres puntos, los cuales se corresponden en su traducción al español con $\mathrm{H}$ (momento del habla), con $\mathrm{R}$ (punto de referencia) y con E (evento). En función de esto, y esgrimiendo la simplificación que aparece en Moreno Burgos (2014: 378), consideramos que las tres relaciones básicas son aquellas que se refieren respectivamente al pasado (E,R-H), al presente (H,R,E) y al futuro (H-R,E). ${ }^{7}$ En estas representaciones, las comas (,) constituyen una relación de simultaneidad, y los guiones (-), una relación de sucesión.

Centrándonos en el tema que nos ocupa, la diferencia entre el PI y el PPC ha sido abordada desde esta perspectiva temporal, como lo refleja el propio estudio de Reichenbach que ya hemos citado: ${ }^{8}$ este estudioso considera que a la forma simple le corresponde una estructura temporal del tipo (E,R-H), mientras que a la compuesta otra del tipo (E-H,R). No obstante, esta posición nos resulta problemática, ya que entra directamente en conflicto con la teoría de la que parte: si desvinculamos a un evento (E) de su punto de referencia (R) estamos eliminando de golpe sus propiedades deícticas, dado que imposibilita que este pueda ser situado en el tiempo. Según parece, lo que Reichenbach (1947) pretendió con

\footnotetext{
námicas), pero no es en rigor aplicable a los estados, que constituyen predicados indivisibles; como tales, no se puede seleccionar ninguna parte de ellos.

${ }^{7}$ En el citado trabajo optamos por sustituir la inicial E (evento) por la inicial S (situación), dado que esta posee un carácter menos restrictivo que la anterior: incluye tanto a los eventos como a los estados. Es necesario no perder de vista esta consideración, a pesar de que mantengamos las convenciones reichenbachianas por claridad expositiva.

${ }^{8}$ Véase también Martínez-Atienza (2008: 218).
} 
ello fue dar cuenta de la semántica del present perfect inglés, el cual se emplea para aludir a situaciones que siguen vigentes en el momento del habla. Sin embargo, siguiendo a Comrie (1976: 60) esto encaja mejor bajo la etiqueta aspectual de Perfecto Continuativo o Persistente: We've lived here for ten years ('Vivimos aquí desde hace diez años'). ${ }^{9}$

Por esta razón, y para paliar estas carencias, otros autores indican que se trata de una cuestión de pertinencia psicológica; esto es, pragmática (cf. Comrie, 1976; Harris, 1982; Detges, 2006; Soto y Zenteno, 2010). No obstante, esta postura tampoco parece ser la adecuada: si la elección se debiera a cuestiones subjetivas, en la cual cada individuo es libre de emplear una forma u otra, quedaría sin explicar por qué se registran tendencias unánimes en cuanto a usos determinados por la procedencia geográfica. Dicho de otro modo: aunque se puedan reconocer contextos en los cuales este pudiera ser relevante, no se trata sin embargo de ningún argumento de validez general — ya que esto queda únicamente restringido a dichos contextos.

En nuestra opinión, una caracterización adecuada de las citadas formas requiere considerar la complejidad del fenómeno en su totalidad, lo cual supone admitir que no debe ser esperable que el español se hable de manera completamente uniforme en una comunidad lingüística tan amplia como es la hispánica. A pesar de que se trata de una constatación obvia, el hecho de que este criterio no se imponga en la bibliografía de manera más rotunda parece deberse a que aún persiste la vieja creencia de que todo lo que

\footnotetext{
${ }^{9}$ Las traducciones en este artículo son nuestras.
} 
no se adapta a una norma estándar debe ser considerado como incorrecto. ${ }^{10}$ Defenderemos por tanto que la diferente distribución del PI y el PPC responde a estadios evolutivos dispares. Esto es, en el español estándar peninsular la segunda de estas formas posee un significado polisémico, ya que puede expresar tanto aspecto Perfecto como aspecto Aoristo. Esta peculiaridad está descartada en las variedades de Latinoamérica, dado que estas remiten únicamente al Perfecto. $^{11}$

\subsection{Semántica de Perfecto y de pretérito}

Según lo expuesto mediante la teoría de Klein (1992), la construcción <haber + infinitivo $>$ se emplea para focalizar una fase inmediatamente posterior a un evento. Esto es lo que observamos en el siguiente ejemplo:

(2) Estoy tendido viendo en la oscuridad los hilos de luz que siguen brillando en mis ojos aunque la linterna se ha apagado y espero como una res a que vengan a matarme [CREA: España].

\footnotetext{
${ }^{10}$ Esta tendencia se ve reforzada por la publicación de obras de orientación normativa, las cuales gozan de gran éxito entre el público en general: Gómez Torrego (2003), Seco (2005), Instituto Cervantes (2013).

${ }^{11}$ Recordemos que en este trabajo sólo nos referimos a los datos México. No nos podemos detener aquí en otras variedades de Latinoamérica, pero para más detalles emplazamos al lector a los trabajos de Flórez (1963), Berschin (1976), Cartagena (1999), Rodríguez Louro (2009), Henderson (2010) y RAE y AALE (2010: 438). Moreno de Alba (2006) explica que, en contra de la tendencia general del continente americano, en las regiones andinas se registra una preferencia por la forma compuesta.
} 
Mediante esta oración se está expresando que en el momento del habla la linterna "está apagada". Esto es, se trata de una construcción estativa, designada más concretamente como un Perfecto Resultativo. ${ }^{12}$ Esta interpretación de las formas compuestas puede llegar por dos vías: mediante una determinada clase accional o más explícitamente mediante los adverbios ya y todavía no. Los verbos que como apagarse pueden expresar nuevos "estados de cosas" son denomidados télicos. ${ }^{13}$ Sin embargo, es necesario considerar que dicho evento no está realmente predicado, sino que pertenece al terreno de la presuposición: "si ahora está apagada, es porque se ha apagado".

Es importante señalar que el PPC ha experimentado un proceso evolutivo que, partiendo de los valores aspectuales aludidos, le permite expresar asimismo tiempo pretérito. Esto ocurre en presencia del complemento adverbial correspondiente (p.e. La linterna se ha apagado hace dos horas) y viene a dar cuenta de una tendencia universal ya referida por Dik (1987). Nosotros consideramos que en la gramaticalización de esta forma se produce una reinterpretación semántica o reanálisis, ${ }^{14}$ mediante el cual se explicita el evento presupuesto por medio de la predicación. Así, partiendo

\footnotetext{
${ }^{12}$ Esto se aprecia de un modo más visible en el caso del pretérito pluscuamperfecto, el cual también puede expresar aspecto Perfecto. Así, la oración A las tres ya se había ido Juan sirve para indicar, no que las tres fuera el momento de la marcha del sujeto de la predicación, sino que a esa hora este no estaba ahí.

${ }^{13}$ Véase Vendler (1957). Empleamos la expresión "estado de cosas" de una manera diferente a como lo hace Dik (1997). Para nosotros los diferentes predicados se pueden dividir en dos: situaciones dinámicas (eventos) y situaciones estáticas (estados o estados de cosas).

${ }^{14}$ Para esta noción véase, por ejemplo, Haspelmath (1998), Detges (1999), Lang y Neumann-Holzschuh (1999) y Hopper y Traugott (2003). Harris (1982) opina
} 
de una oración como Juan ya ha llegado, encontramos en Moreno Burgos (2014: 173) la siguiente representación:

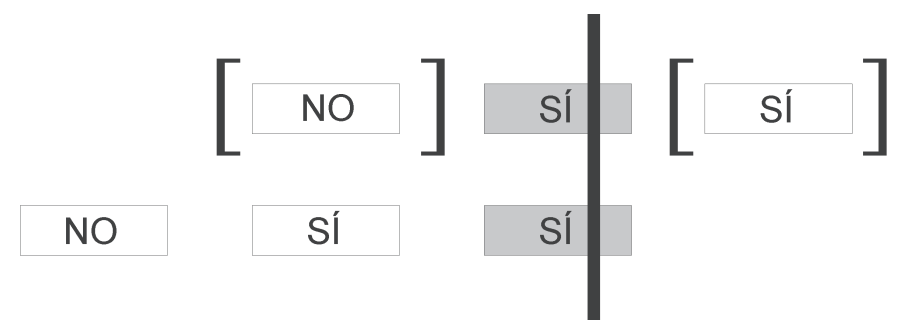

Figura 1. La expresión del pretérito

Como observamos, el adverbio fasal ya introduce una fase afirmada (Juan está aquî), al tiempo que una presuposición (Juan no estaba aqui) y una expectativa (Juan estará aqui)..$^{15}$ Pues bien, lo que hace el hablante es considerar que la segunda de las fases afirmadas es el verdadero resultado de un evento, el cual aparece caracterizado desde el signo de la telicidad (esquema no-si). En una segunda instancia, deja de ser relevante el estado de cosas en el presente, pasando a ser ocupado por un complemento temporal: p.e. Juan ha llegado esta semana. Es necesario indicar que a esta semántica de pretérito le corresponde un significado aspectual de Aoristo.

Esta evolución no se registra en todo el dominio hispánico, como es el caso de Canarias, Asturias, León, Galicia o en Latinomérica. ${ }^{16}$ Observemos los siguientes ejemplos:

que el factor causante del proceso de gramaticalización se basa más bien en la pertinencia psicológica.

${ }^{15}$ Para más detalles véanse los trabajos de Muller (1975) y Garrido (1992).

${ }^{16}$ Cf. Lope Blanch (1961), Berschin (1976), Cartagena (1999) y Kempas (2008a). 
(3) Joe Torre estaba tan emocionado ayer que abandonó el "Yankee Stadium" con su sucio y sudado uniforme de los Yanquis aún puesto. Y hoy estuvo tan ocupado que no contestó las dos primeras llamadas del presidente Clinton para felicitarlo [CREA: México].

(4) En esta profesión subes y bajas, y no puedes olvidarlo. Por cada día maravilloso que pasas tienes que decirte: hoy ha estado bien, pero mañana todo puede ser horrible [CREA: España].

En la variedad estándar peninsular rige una regla que puede ser formulada de la siguiente manera: en sus usos temporales (esto es, con interpretación aspectual de Aoristo), el PPC aparece con complementos hodiernales; es decir, con aquellos que incluyen el momento del habla. En el caso de que aparezcan complementos prehodiernales, se emplea el PI. Esta regla no se cumple en México, tal y como observamos en el ejemplo aducido. Nuestra tesis es considerar que los casos como los de (4) reflejan un proceso de gramaticalización más avanzado que el de (3), mediante el cual se prevé que la forma compuesta pase a sustituir completamente a la simple tal y como se atestigua en francés o en italiano. En apoyo a esta hipótesis tenemos enunciados como los que cita Kempas (2008b: 235), en los que la forma he ido aparece incluso cuando se tienen complementos hodiernales:

(5) Ayer he ido al cine.

(6) He ido al cine la semana pasada.

Estos datos no vendrían más que a poner en evidencia una tendencia lógica en la reorganización del sistema lingüís- 
tico, lo cual implica a su vez la desaparición de los elementos redundantes. Esta constatación puede resultar inverosímil a oídos de un hablante latinoamericano, pero adquiere verdadero sentido cuando se piensa que el español peninsular estándar constituye a este respecto la variedad del español más innovadora. Al mismo tiempo, nuestra hipótesis viene a ser la confirmación de la línea avanzada por Lope Blanch, la cual no ha alcanzado por desgracia la unanimidad necesaria que una observación de este rango merece. En palabras del propio autor:

No se trata tampoco en este caso de que la norma culta mexicana se haya alejado de la española o hispánica general, sino de diferente evolución, castellana y mexicana, a partir de un estado de cosas aún no bien delimitado dentro de la norma lingüística existente en el siglo XVI, cuando las formas canté y he cantado no había alcanzado aún una distribución bien definida de sus funciones respectivas (1992: 1225). ${ }^{17}$

A pesar de que en los ejemplos anteriores no se registra, existen también oraciones en las que el PPC no aparece acompañado de ningún complemento adverbial de ningún tipo (ni hodiernal ni fasal). Es por esto que proliferan las teorías sobre el componente pragmático. Observemos la siguiente oración:

\footnotetext{
${ }^{17}$ Esta observación remite a su vez a otro trabajo anterior: Lope Blanch (1961). Para una reflexión en este sentido véase Laca (2009), así como Harris (1982) y Detges (2006).
} 
(7) Las autoridades etíopes han dicho que unos 150 soldados resultaron muertos y otros 250 heridos en el combate que fue iniciado con el ataque con morteros y cohetes [CREA: España].

En ejemplos como este, los lingüistas especializados han centrado su interés en dilucidar el porqué del uso de la forma han dicho en detrimento de dijeron. Antes de dar una respuesta, debemos reiterar que es esencial distinguir entre la variedad del español utilizada, ya que en función de esto los análisis semánticos resultan ser por tanto distintos. Con respecto a oraciones como esta, indican estudiosos de la talla de Alarcos Llorach (1947: 122-127) que el PPC constituye un "presente ampliado", el cual depende de factores subjetivos. En palabras de este autor, "el perfecto compuesto indica muchas veces una acción cuyos resultados duran todavía en el momento en que se habla”. Desde nuestro punto de vista, existen sin embargo varias razones para poner en duda esta formulación en esos términos.

En primer lugar, porque así formulado resulta difícil comprender en qué medida se puede hablar de un resultado con respecto a un evento atélico como decir. Además, esta cuestión, que suele ser referida con respecto al español, parece surgir porque se identifica erróneamente la semántica del español con la del inglés. Esto es, con la consabida interpretación de Continuativo del present perfect. ${ }^{18}$

\footnotetext{
${ }^{18}$ Alarcos Llorach (1947: 112) cita a Keniston (1937: 445) para describir el PPC del español: "from the point of view of the speaker or writer the action or state in question must extend to a point which falls within his present interest".
} 
Por otro lado, el criterio de la subjetividad sólo se debería aplicar a aquellos enunciados en los que no aparecen complementos temporales. En los casos en los que sí aparecen no podemos hablar por tanto de criterios interpretativos, ya que la alternancia en el uso del PPC y el PI en el español estándar peninsular se exige por el criterio de la hodiernalidad referido arriba; esto es, está relacionado directamente con la semántica de los complementos.

Únicamente a partir del criterio evolutivo que aquí defendemos podemos cubrir la totalidad de los contextos (con o sin complementos), sin necesidad de considerar la pertinencia psicológica como el factor primario. ¿Cuál es por tanto el motivo de la elección de la forma compuesta en ejemplos como el de (6)? Nuestra tesis es considerar que en el español estándar peninsular el PPC representa la forma que se emplea por defecto (cf. Schwenter y Torres Cacoullos, 2008: 33), quedando el PI como la forma marcada. ${ }^{19}$ Si pensamos en la secuencia Velázquez [ha pintado/ pintó] Las Meninas, llegamos a la conclusión de que el hablante puede elegir entre la lectura de Perfecto y la de Aoristo: la preferencia por pintó implica el empleo de una forma que está destinada a desaparecer, la cual desencadena a su vez una lectura pragmática de lejanía. A partir de ahí, se extiende este fenómeno también a los predicados atélicos, tal y como hemos observado en (7). No obstante no cree-

19 Para los conceptos de "marcado" y "no marcado" véanse Lamíquiz (1998: 3637) y Comrie (1976: 111-122). Esta apreciación conlleva a su vez lo siguiente: si el PPC deja de ser relevante en la expresión del Perfecto, lo lógico es que esta noción pase a ser vehiculada de manera preferente a partir de otra forma; un candidato idóneo para ello sería la perífrasis < acabar de + infinitivo $>$. 
mos que el proceso gramaticalizador haya surgido en estos contextos desprovistos de complementos; si esto fuera así, no se podría justificar la alternancia entre los hodiernales y los prehodiernales, ya que como hemos visto en (5) y (6) la tendencia va camino de una generalización de los contextos en los que la forma compuesta aparece, no de su restricción.

\section{El PPC en su lectura aspectual}

\subsection{Maneras de expresar estatividad}

En las líneas que siguen detallaremos cómo se conceptualiza la estatividad en el uso del PPC en Latinoamérica. Nuestra tesis pasa por defender que constituye una lectura particular del Perfecto Resultativo que no se da en el español estándar peninsular. Consideramos que esta lectura está vinculada con la semántica <estar + gerundio >, la cual no sólo expresa valores aspectuales, sino también valores modales. Antes que nada nos centraremos en detallar esto último.

Partiendo de la descripción de Klein (1992) hemos considerado que el aspecto Imperfecto es aquel que selecciona una fase interna de un evento; esto es, se llega a la conclusión de que el evento se encuentra en desarrollo. Pues bien, una descripción en tales términos remite sobre todo al llamado Imperfecto Progresivo, pero requiere una explicación más detallada en el caso del Continuo (El teléfono sonaba constantemente) y el Habitual (Iba al cine a menudo), las otras subvariedades con las que se vincula al aspecto Imper- 
fecto. ${ }^{20}$ Para nuestro análisis sólo serán relevantes ejemplos como los siguientes:

(8) Cuando volví a su lado, el borracho estaba llorando porque no era justo que a una dama, como se notaba a la legua que era mi madre, le pasaran esas cosas tan horribles [CREA: México].

(9) Víctor empezó a acariciarle el pelo sin decir nada y estuvieron un rato abrazados. Luisa tardó en darse cuenta de que él también estaba llorando, sólo lo supo cuando le oyó, por fin, la voz [CREA: España].

Como observamos, el Tiempo del Foco selecciona una parte interna del Tiempo de la Situación, el evento atélico llorar. Esto puede ser descrito metodológicamente de la siguiente manera: se trata del fotograma de una película, considerando que el fotograma posee un carácter estativo, y la película, eventivo. Esta observación, que proviene de la teoría subeventiva de Moreno Cabrera (2003) y Pustejovsky (1991), no puede ser sin embargo aplicada al aspecto Aoristo, ya que este no da acceso a la estructura interna de los predicados dinámicos (cf. Moreno Burgos, 2014: 347-352). Es importante señalar la similitud que existe entre los predicados como Estar en casa y las fases seleccionadas por el Tiempo del Foco: en ambos casos se trata de estados.

\footnotetext{
${ }^{20}$ Esta subclasificación aparece en Bertinetto (1986) o García Fernández (2004). No entraremos aquí en detalles; para ello véase el trabajo de Moreno Burgos (2014).
} 
Debemos llamar la atención sobre lo siguiente: ni en (8) ni en (9) se puede saber si la situación designada mediante el predicado llorar sigue vigente en el momento del habla: puede ser que sí o puede ser que no. Esto contrasta con estos otros ejemplos:

(10) Yo estuve analizando los versos durante cinco minutos, y llegué a la conclusión de que sí nos servían. Nos levantamos. Intercambiamos nuestros besos y nuestros malos alientos. Estefanía preparó el desayuno. Nos sentamos a la mesa [CREA: México].

(11) Francesco Moser no sabía si quedarse o marcharse [...]. Así que se sentó en una caja de botes de spray y le estuvo viendo trabajar un rato en silencio. Luego empezó a hablar [CREA: España].

La cuestión que despierta el interés de los lingüistas es el hecho de que el auxiliar de la perífrasis progresiva no sólo aparece en pretérito imperfecto (o en presente), sino también combinado con formas perfectivas como el PI. Ya ha sido señalado por Bertinetto (2000) o Squartini (1998) que esta situación no se da en otras lenguas románicas como el italiano, lo cual revelaría una etapa específica en el desarrollo evolutivo de la construcción española. La pregunta que nos debemos hacer es si en estos casos la estructura completa expresa una variedad que no sea la imperfectiva, como afirman García Fernández (2006: 136) o Sánchez Prieto (2011: 146). ${ }^{21}$

\footnotetext{
${ }^{21}$ El primer autor considera que a una oración como Ha estado durmiendo hasta ahora y necesita dormir más le corresponde la semántica del Perfecto Continuativo; sin embargo, ya hemos indicado que consideramos erróneo aplicar esto al
} 
Nosotros creemos que no, y que es necesario detallar la manera en la cual se conceptualiza la perfectividad en el caso de los estados. Así, en ejemplos como Ayer estuve en Madrid, se considera que una situación que era verdad en un momento concreto del pasado, ha dejado de serlo en el momento del habla. ${ }^{22}$ Esto lo podemos ejemplificar mediante el adverbio fasal ya no: ayer estaba en Madrid, ahora no lo está. Este mismo esquema es aplicable a la perífrasis de Progresivo:

- Antes estaba analizando los versos; ahora no estoy analizando los versos.

- Antes lo estaba viendo trabajar; ahora no lo está viendo trabajar.

Tal y como indica Bertinetto (2000), las oraciones como (10) y (11) se interpretan como durativas; sin embargo, consideramos que esta es una lectura pragmática que surge al considerar dos puntos de referencia: por un lado, el que le corresponde a la perífrasis progresiva con el auxiliar en pretérito imperfecto (estaba analizando, estaba viendo) y, por otro, el del momento del habla (no estoy analizando, no estoy viendo). En lo que sigue, y sin perder de vista esta consideración, nos referiremos a dichos ejemplos bajo la etiqueta de durPROG.

Por otro lado, encontramos frecuentemente en la bibliografía especializada que la perífrasis de Progresivo no es compatible con los estados. Esta regla confirmaría el hecho de que los estados no son dinámicos y que, por tanto, no

español. El segundo autor vincula la progresividad con la perfectividad, lo cual constituye una dificultad teórica todavía mayor.

22 Para más detalles véase Moreno Burgos (2014: 265-269). 
se puede seleccionar ninguna de sus fases. A pesar de todo, constatamos que oraciones como las siguientes no son ni mucho menos una excepción:

(12) La mayor parte del crecimiento de la inversión crediticia correspondió al sector hipotecario, en donde destacan los 31.862 millones de pesetas captados en hipotecas en Madrid, producto que está siendo la base de la expansión de la entidad en dicha comunidad autónoma [CREA: España].

(13) Las condiciones de infraestructura también están siendo propicias, aun cuando son susceptibles de perfeccionarse porque hay muchos factores de carácter regulatorio, que contrario a lo que sucede en otros países, aquí están impidiendo su desarrollo [CREA: México].

Por esta razón, si queremos seguir considerando este argumento como válido, debemos realizar una serie de matizaciones. Autores como Bertinetto (2004: 294) indican que en ocasiones se llega a una lectura agentiva. En efecto, esto podría ser aplicable a una oración como Juan está siendo tonto esta noche, ${ }^{23}$ sin embargo no constituiría una explicación adecuada de los ejemplos que nosotros ofrecemos. En Moreno Burgos (2014: 315-321) se argumenta que se trata de una perífrasis homónima que se emplea como recurso para reducir el contenido de verdad de las predicaciones estativas. Esto es, vivir en una ciudad $x$ implica ser habitante de dicha ciudad, mientras que estar viviendo en una ciudad

\footnotetext{
${ }^{23} \mathrm{El}$ autor se basa originalmente en datos del inglés: John is being silly tonight.
} 
$x$ pone precisamente en duda esta aseveración: se puede tratar únicamente de una persona que está de paso; lo mismo se puede aplicar a otras predicaciones como Juan está trabajando en Bimbo o Juan está llegando últimamente tarde a clase, las cuales suponen formulaciones menos contundentes que Juan trabaja en Bimbo y Juan llega últimamente tarde a clase, respectivamente.

En el siguiente apartado mostraremos que la semántica del PPC en Latinoamérica obedece, en la mayoría de los casos, a una predicación como las de (10) y (11) en la que, a su vez, la fase negada en el presente es interpretada desde la modalidad. El hecho de que se considere la validez de la situación en el momento del habla estaría supeditado a que se suspenda la restricción en torno al compromiso de verdad de la aseveración. Para tener acceso a esta interpretación se hace necesaria, sin embargo, la presencia de un complemento de duración o que remita de alguna manera a la iteración de la situación dada.

\subsection{El caso de Latinoamérica}

Antes que nada, cabe recordar que el punto de partida de nuestro análisis debe ser formulado de la siguiente manera: en el español de México el uso del PPC revela características del aspecto Perfecto, pero no del Aoristo. No se trata exactamente de lo que encontramos en oraciones como La linterna se ha apagado, sino de una variante iterada del PPC. ${ }^{24}$

${ }^{24}$ En Moreno Burgos (2014: 166-171) se explica más concretamente que la evolución del PPC en el español peninsular estándar es cualitativa (de aspecto 
Esta observación, que ya aparece en Lope Blanch (1961), la interpretaremos nosotros de la siguiente manera: en lugar de contemplar un único estado de cosas, se consideran dos. Esto es, en el plano aspectual, el PPC representa en México una tendencia innovadora con respecto al español estándar peninsular. ${ }^{25}$

El contraste entre el uso de los adverbios fasales ya y todavía no supone un buen ejemplo: mientras que el primero aparece normalmente con la forma simple, el segundo suele ir acompañado de la forma compuesta. Esta constatación, que aparece en Flórez (1963: 279) con respecto a Colombia, también se puede observar en las siguientes oraciones:

(14) No ha llegado todavía al estrecho de Magallanes la televisión, lo que, al igual que en las Malvinas, debe considerarse un beneficio. Los puntarenenses leen mucho, sobre todo en las largas noches invernales [CREA: México].

(15) David Konzevik, uno de los economistas latinoamericanos de mayor prestigio, y de los más exitosos conferencistas en México, repetirá el año próximo una serie de conferencias que ofreció,

\footnotetext{
a tiempo), mientras que en Latinoamérica es cuantitativa (se conceptualizan varias fases).

${ }^{25}$ Descartamos que esta lectura del PPC se haya podido dar en la variedad europea del español antiguo. Tenemos diferentes argumentos para basarnos en ello. En primer lugar el criterio de la unidireccionalidad del cambio (cf. Haspelmath, 2004), con la dificultad de demostrar que el proceso de gramaticalización se haya revertido. En segundo lugar, la comparación con el portugués (cf. Berschin, 1976: 42-44), lengua donde se privilegia el uso de una forma simple de pretérito. El retroceso de la forma compuesta parece estar directamente relacionado con el surgimiento de una perífrasis iterativa: Tem feito calor últimamente ('Ha estado [y está] haciendo calor últimamente').
} 
totalmente gratuitas, a jóvenes entre 17 y 25 años, con el título "El futuro ya llegó: ¿está Usted preparado"? [CREA: México].

En efecto, la primera de ellas representa un uso similar al del español estándar peninsular. El empleo del PPC estaría justificado porque se produce una iteración de un estado de cosas: "antes no estaba allí, ahora no está allí". Al mismo tiempo, como indica Lope Blanch (1961) se contempla una expectativa ("estará aquí) que no es accesible a partir de la negación del PI (no llegó). En el polo opuesto se encuentra ya llegó, debido a que no permite el uso de la forma compuesta tal y como hemos observado en el español europeo. Eso sería un síntoma de que, según leemos en Rodríguez Louro (2009: 158, 203), el PPC ha sufrido un retroceso en ciertos contextos. Ahora bien, en contra de lo que indica la autora, esto no concierne al valor de pretérito (que en nuestra opinión nunca se ha dado en el área latinoamericana), sino al valor aspectual: el PPC ha dejado de expresar el Perfecto Resultativo "simple", para especializarse en contextos iterados. ${ }^{26}$ Veamos ahora estos otros ejemplos:

(16) Esta semana se ha trabajado en el asunto, aunque hoy es feriado en EE.UU. La siguiente semana se reanudarán las evaluaciones

\footnotetext{
${ }^{26}$ Rodríguez Louro (2009: 30) rechaza la tesis de Lope Blanch (1961) sobre la gramaticalización del PPC y que nosotros defendemos aquí. Se basa en argumentos como el hecho de que el PPC en español rioplatense admitía en el siglo XIX complementos prehodiernales (2009: 170-171). Sin embargo, la argumentación no es la correcta, ya que dicha autora considera que la presencia de adverbios como ayer constituye una prueba de la perfectividad del PPC, algo que puede ser rápidamente refutado: Ayer a las tres estaba en mi casa.
} 
y hay incluso probabilidades de que se pudiera elegir a un comprador de esa compañía [CREA: México].

(17) Entiendo al deportista y muchas veces no dice lo que quiere decir, está presionado, preocupado, y a veces expresa cosas que no siente en realidad. Conozco a Dionicio, he platicado varias veces con él y estoy seguro de que no lo han entendido bien. Los periodistas lo criticaron mucho cuando llegó molesto de Gotemburgo por haber sacado segundo lugar [CREA: México].

En (16) se predica lo siguiente: se trabajó y se trabaja en el asunto, lo cual aparece sintetizado mediante el PPC. Esto es, la forma ha trabajado no expresa una lectura temporal con interpretación de Aoristo, sino que se trata más bien del aspecto Perfecto: en el momento del habla se predica en términos estativos sobre una situación que ya se dio en el pasado. Expliquémoslo poco a poco.

Si consideramos que el predicado trabajar debe aparecer bajo el signo de la perfectividad en un instante que precede al momento del habla surgiría la siguiente paradoja: estaríamos pensando en un evento pero no en un estado (y no se cumpliría, en consecuencia, la condición impuesta por el Perfecto). Ahora bien, este problema se solventa fácilmente al sustituir se trabajó por la perífrasis se estuvo trabajando, ya que desde el esquema mostrado arriba, asignamos fases estativas en el desarrollo interno del evento: antes se estaba trabajando, pero ahora mismo no se está trabajando. El hecho de negar una fase en relación con el momento del habla no supone incurrir en ninguna contradicción con lo expuesto hasta ahora: se puede decir que "alguien está trabajando en 
un asunto", aunque en el instante de la predicación no se esté ejecutando ninguna acción (por tratarse, por ejemplo, de un día feriado). Por esta razón, consideramos que lo que hace un hablante mexicano como el de (16) es cancelar el estado negativo perteneciente al momento del habla y, en su lugar, introducir el valor modal de la perífrasis <estar + gerundio $>$, lo cual bloquea la perfectividad. Lo mismo se puede decir con respecto a (17): he platicado varias veces con él supone que el sujeto de la predicación estuvo platicando; esto es, que en varios momentos del pasado estaba platicando. En el momento del habla no se está desarrollando la acción referida, pero esto no impide que a partir de la información anterior se pueda construir una oración estativa: Estoy platicando con él (en el sentido de que "estoy en contacto con él"). ${ }^{27}$ Lo expresamos gráficamente de la siguiente manera:

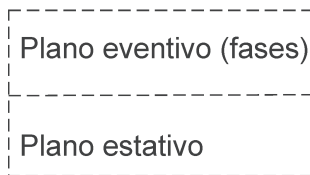

$$
\text { Se estaba trabajando }
$$

No se está trabajando

Se está trabajando

Figura 2. El Perfecto en Latinoamérica

Una prueba de que el PPC en Latinoamérica parte de la estructura durPROG reside en el hecho de que sólo las formas perfectivas (y no las imperfectivas) admiten comple-

\footnotetext{
${ }^{27}$ Este esquema también es aplicable a los estados léxicos. Pensemos por ejemplo en la oración ¿Cómo has estado? del español mexicano, ahí donde el europeo prefiere ¿Cómo estás? En el uso americano la pregunta tiene un carácter más extensivo, ya que abarca tanto el pasado (“¿Cómo estuviste?”) como el presente (“¿Cómo estás?”).
} 
mentos durativos como los que se aprecian en el siguiente ejemplo:

(18) Cada vez es más claro que para cruzar el umbral del Estado de derecho, de la democracia ciudadana, de la independencia de los distintos poderes, es necesario pasar por la sustitución generalizada de la oligarquía política que nos ha gobernado durante cerca de siete décadas [CREA: México].

Esto se debe a que los complementos introducidos por durante se caracterizan por no incluir el momento del habla, de manera que sólo es aceptable una secuencia como Estuvo gobernando durante siete décadas, al contrario de lo que ocurre con Estaba gobernando durante siete décadas, que resulta anómala. A esta interpretación perfectiva de (18) se le añade al mismo tiempo una lectura mediante la cual se considera que en el momento de habla también "está gobernando". Esta información contrasta, sin embargo, con el español estándar peninsular: el ejemplo propuesto, pronunciado por un habitante de Madrid, significaría que el sujeto de la predicación ya no está gobernando.

No suscribimos, por tanto, las ideas de Soto y Zenteno (2010), quienes analizan un discurso de la presidenta de Chile para llegar a la conclusión de que el empleo de esta forma se fundamenta a partir de criterios de subjetivación, basándose en ejemplos como Nuestra economía ha crecido a tasas extraordinarias. El error de base consiste en considerar las oraciones aisladamente, ya que como se extrae del propio discurso (Bachelet Jeria, 2007), la presidenta alude explícitamente a un periodo de tiempo determinado: "el 
progreso que hemos experimentado en estos años de democracia es enorme". Lo mismo cabe decir de la oración He puesto toda mi fuerza para llevar adelante el proyecto de progreso y justicia social que la mayoría de los chilenos anhe$l a$, dado que justo antes se indica: "Hace un año hablé del Chile de todos que estamos construyendo". Indudablemente se piensa en la duración de un proyecto que ya estaba en marcha un año antes al momento del discurso.

Si echamos de nuevo un vistazo a la oración (16), observamos que el enunciado aparece acompañado por un complemento como esta semana, lo cual implica, como en el caso de todavía no, una lectura iterativa (el lunes estaban trabajando, el martes estaban trabajando, etc.). En la oración (17), por su parte, aparece el complemento varias veces, mientras que en el discurso de la presidente chilena se habla de estos años de democracia.

Al margen de los complementos temporales, otro factor que contribuye a esta lectura iterativa son los casos en los que un determinado elemento oracional se encuentra en plural:

(19) Para mi sorpresa, hasta julio de 1996 no se ha hecho ninguna bonificación y, por el contrario, cada mes me han llegado avisos de cobro para que pague mi "adeudo", incluyendo desorbitados intereses, gastos de cobranza e IVA [CREA: México].

(20) Cada vez que me llama dejo que hable él y aprendo mil agradables o desagradables cosas. Los temas que ahora han salido a luz son casi los mismos que traté el lunes pasado en Monitor de Radio Red: algunos aspectos del llamado "milenarismo" [CREA: México]. 
En relación con estos datos, mencionaremos la posibilidad apuntada por Martínez-Atienza (2008: 224) de que el Perfecto en Latinoamérica esté relacionado con el valor de Continuativo del present perfect inglés. Recordemos el ejemplo de Comrie (1976: 60) presentado arriba: We've lived here for ten years ('Vivimos aquí desde hace diez años'). Pues bien, la correspondencia en ambas lenguas no es exacta, dado que la forma inglesa exige la presencia de los complementos since o for ('desde' y 'desde hace', respectivamente); mientras que, en el caso de Latinoamérica, ya hemos visto que no es un requisito obligatorio. Comparemos ahora los siguientes ejemplos:

(21) Mi familia ha hecho negocios en México desde hace 50 años. Por ejemplo: en estos 50 años mi familia ha tenido empresas, plantas de cemento, lanchas para esquiar y botes para pescar, taxis, coches de renta [CREA: México].

(22) La EET de ovejas y cabras (scrapie) es la enfermedad relacionada con priones prototípica. Esta se conoce desde hace más de 250 años como una enfermedad enzoótica. En el Reino Unido de 0.5 a $1 \%$ de las ovejas presentan esta condición cada año [CREA: México].

Aquí vemos que la primera oración aparece con el PPC, pero no la segunda. Esto es, dado que se trata de un contexto restringido, las investigaciones deberían ir más bien orientadas a dilucidar el porqué de la alternancia entre el presente y el PPC en presencia de desde (hace), algo que no analizaremos aquí. 


\section{Lecturas modales}

\subsection{El Perfecto Experiencial}

En las líneas anteriores hemos dejado constancia de que el aspecto Perfecto es subclasificado en Resultativo y Continuativo. La primera lectura da cuenta de una manera canónica de las propiedades que le hemos atribuido a esta variedad aspectual; con respecto a la segunda, acabamos de ver, que puede ser aplicada al inglés, pero que no hay razones claras para hacerlo con el español. La bibliografía señala que existe una tercera subdiferenciación, que se ha denominado Perfecto Experiencial (cf. Bertinetto, 1986; Comrie, 1976; Fenn, 1987). Pues bien, en este apartado sostendremos que esta subclase surge al realizarse una lectura modal del Resultativo.

Mediante la etiqueta de Experiencial, los diversos autores ponen de manifiesto que el PPC se emplea para referirse a situaciones que se pueden adscribir a la experiencia personal de un individuo. Desde nuestro punto de vista, existen unos requisitos para que se dé esta lectura; uno de ellos es la presencia del adverbio $y a$ :

(23) Ferreri ha estado ya en Canarias para elegir los exteriores de su nueva película, una coproducción italo-española. La naturaleza volcánica de Lanzarote y el escenario singular de las Cañadas del Teide (Tenerife) han sacado de dudas al realizador [CREA: España].

En efecto, en este caso estamos indicando que el sujeto de la predicación ha visitado al menos una vez en su 
vida las islas Canarias. Demos preguntarnos, sin embargo, lo siguiente: si los adverbios como ya dejan al descubierto una semántica fasal, ¿qué es lo que añade a la semántica de predicados como estar que ya de por sí son estados y, por tanto, indivisibles? Nuestra tesis es defender que en este caso se trata más bien de un mecanismo tematizador, mediante el cual se considera que se trata de una información conocida. ${ }^{28}$ Esto es, un enunciado como Ferreri ha estado en Canarias, exento de todo complemento temporal, remite al aspecto Aoristo, el cual también se expresa mediante el PI: Ferreri estuvo en Canarias. Ahora bien, la presencia de ya hace presuponer al oyente que esta información no es nueva.

Con esto podemos llegar a la siguiente conclusión: la combinación de ya con el Aoristo provoca una lectura temática, mientras que en combinación con el Perfecto da lugar a una lectura fasal. Esta última surge cuando los predicados télicos aparecen con el PPC: Juan ya ha llegado significa que antes no estaba aquí, pero que ahora sí. El hecho de que podamos encontrar oraciones en el español estándar peninsular como Ya he comido se debe al proceso de gramaticalización aludido, mediante el cual la forma compuesta pasa a adoptar la función aorística de la simple. En este caso, dicha oración remitiría igualmente a una información conocida. Ahora bien, dada la confluencia homonímica de las formas, Ya he comido pasa a reinterpretarse desde un punto de vista aspectual (Perfecto Resultativo), en una lectura ter-

${ }^{28}$ Para más detalles sobre estos conceptos véase RAE y AALE (2010: cap. 40). 
minativa: "he acabado de comer". Esto se puede desglosar fasalmente de la siguiente manera:

- Estaba comiendo

— No estoy comiendo

— No estaré comiendo

La interpretación de Perfecto Experiencial surge precisamente a partir de esta lectura resultativa. Si decimos Yo ya he comido caviar, la experiencia de "comer caviar" no sólo será cierta en el momento del habla (en una fase inmediatamente posterior al estado afirmado), sino también en cualquier otro momento del futuro. De ello se derivan dos componentes importantes: uno modal, ya que remite a la eventualidad de momentos posteriores al momento del habla; otro pragmático, dado que el evento debe ser pragmáticamente relevante. Para visualizarlo mejor, nos serviremos de la representación que aparece en Moreno Burgos (2014: 363):

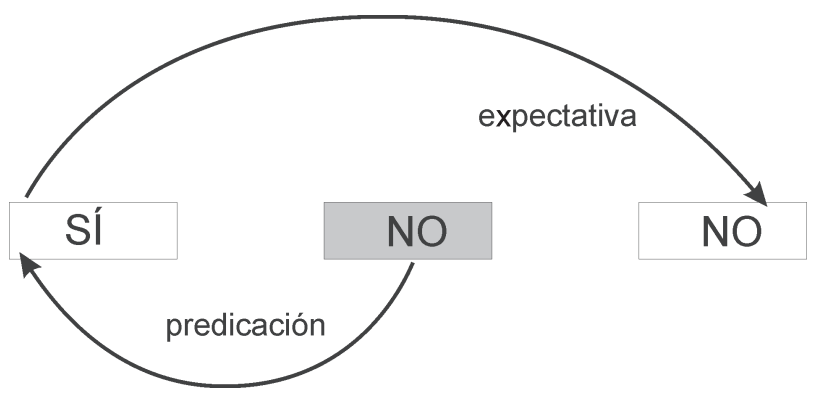

Figura 3. Perfecto Experiencial 
Otro de los requisitos es que aparezca un complemento temporal de frecuencia del tipo $x$ veces. Observemos el contraste entre los siguientes ejemplos:

(24) Según parece, estas filtraciones tienen que ver con el extraño periplo del jefe del Gobierno australiano, Fraser, que esta semana ha visitado dos veces Washington $\mathrm{y}$, entre medias, Bonn, París y Londres [CREA: España].

(25) El amor... No he estado enamorada muchas veces. El amor es tan importante... Es el otro junto a ti. La otra persona... Me he casado cuatro veces. Espero ya terminar mi vida en compañía de Tulio... Pero, desgraciadamente, se planean las cosas y no siempre salen como quisiéramos... [CREA: España].

En el primer caso se trata de una frecuencia que podemos denominar "relativa", ya que esta aparece enmarcada dentro de un periodo de tiempo concreto (y el verbo se interpreta como un tiempo pretérito); sin embargo, el segundo de los ejemplos establece una frecuencia en términos absolutos, ya que como marco se establece la experiencia vital de una persona; esto es, todo lo vivido hasta ahora. Esta descripción permite establecer un esquema similar a lo presentado en la figura 3: el sujeto de la predicación no se está casando en el momento del habla, pero lo estaba haciendo en un momento anterior; en cualquier instante posterior seguirá siendo pertinente esta predicación. Consideramos, por tanto, que la lectura Experiencial a partir de complementos como una vez llega porque estos poseen también aquí una naturaleza temática. Así, decir que alguien ha estado en Canarias supone introducir una 
información nueva; pero si decimos que ha estado una vez en Canarias obtenemos una especie de redundancia entre la información expresada por el predicado en Aoristo y la que se deriva del complemento que la acompaña.

Lo expuesto hasta ahora nos lleva por tanto hasta una hipótesis que sólo dejaremos planteada aquí, pero que no desarrollaremos más en detalle: la lectura Experiencial surge en realidad a partir del tiempo pretérito, el cual permite establecer una lectura fasal derivada. Esto nos sitúa tras la siguiente pista: desde esta perspectiva, la lectura Experiencial no existiría en ámbitos geográficos como el mexicano. Los ejemplos que parecen ser tales muestran una semántica como la descrita en los ejemplos (16) - (20).

Añadiremos finalmente que la negación (25) se realiza a través del adverbio nunca (Nunca me he casado). Observemos ahora los siguientes ejemplos:

(26) La verdad es que nunca he visto La Habana desde el mar. Pero desde la ciudad puede advertiste que las fachadas principales de los edificios no miran tierra adentro sino a la bahía [CREA: México].

(27) Yo también podría creer que no eres quien dices, nunca he visto una cordobesa tan rubia. ¿Cómo has salido tú tan rubia y tu hermana tan morena? [CREA: España].

Del contraste entre ambos parece advertirse que en (26) no se descarta en un futuro ver La Habana desde el mar, mientras que en (27) no existe tal expectativa. No debemos pasar por alto que nunca posee un segundo significado que, a diferencia de lo que ocurre aquí, sirve para negar a los 
correspondientes enunciados en los que aparece siempre. De ello hablaremos seguidamente.

\subsection{El contenido de verdad: siempre}

En este apartado mostraremos que la combinación del adverbio siempre con el PPC se emplea como un recurso para indicar que la información no constituye nada novedoso; esto es, que se trata de información conocida. ${ }^{29}$ La interpretación de las oraciones es sin embargo diferente según la variedad lingüística de la que se parta. Observemos los siguientes ejemplos:

(28) La relación entre Ron Dennis, patrón de la escudería británica que este año monta los motores Mercedes Benz, y el propio Alain Prost siempre ha sido muy buena. En la actualidad, ambos se necesitan mutuamente. Y por ello, han decidido unirse de nuevo para sacar adelante un proyecto común [CREA: España].

(29) Cuando vendí el carretón primero se lo ofrecí a él, pero no lo quiso, estaba más que enojado, así que lo vendí más adelante. $M i$ primo siempre ha sido muy débil de carácter y atenido, nunca se arriesga a nada, ni tan siquiera a hacer cruzas entre las vacas, me duele decirlo pero es la pura verdad [CREA: México].

En el español estándar peninsular, el uso del PPC remite a un pretérito con interpretación de Aoristo. Al emplear una

\footnotetext{
${ }^{29}$ Este es un significado diferente al que tradicionalmente se le atribuye, ya que dicho adverbio sirve además para expresar habitualidad y, en consecuencia, se combina con el aspecto Imperfecto. Así, una oración como Siempre iba al cine los domingos, indica la repetición sin excepciones de un evento.
} 
variedad perfectiva, lo que hace el hablante es restringir el contenido verdad de la proposición al pasado, sin que esta se pueda hacer extensible al presente. Sin embargo, la presencia del adverbio siempre añade una información de carácter mucho más rotundo: no hay momento del pasado en el que esta no sea cierta. Esto es, la información ya tenía que ser conocida anteriormente. Con esto se llega a su vez a una lectura pragmático-modal a partir de la cual se considera que si "la relación era constatemente buena, no tiene más remedio que serlo ahora también".

En el caso del español de Latinoamérica se trata más bien del aspecto Perfecto, un ejemplo similar a lo visto en los apartados precedentes. Aquí la predicación con respecto al momento del habla no llega por mecanismos inferenciales, sino gramaticalmente: el carácter del sujeto de la predicación fue débil en el pasado, algo que también se manifiesta de algún modo en el presente. En este punto tenemos por tanto que disentir de Gutiérrez Araus, quien se expresa de la siguiente manera:

De igual modo, sin un hablante tanto de España, como de América, asegura: Beatriz ha mentido toda su vida quiere decir que el decir mentiras de Beatriz, fenómeno iniciado en un momento pasado, continúa y prevé que seguirá adelante. Sin embargo, si dice: Beatriz mintió toda su vida puede ser que ya no viva Beatriz (su vida pertenece al pasado), o bien que ya no mienta (la acción de mentir pertenece al pasado) (1995: 22-23).

Aquí partimos de la predicación estativa Beatriz miente. No obstante, nosotros nos vemos en la necesidad de hacer 
las siguientes puntualizaciones: el análisis de la forma compuesta no puede ser idéntico en el caso de las variedades peninsulares y las americanas; esto se debe, en parte, a que en la primera de ellas la información relativa al momento del habla no está realmente predicada. Finalmente, hemos de decir que la semántica del PI debe ser enfocada desde los procesos de gramaticalización a los que hemos aludido anteriormente. Si lo aplicamos a nuestros ejemplos, La relación siempre fue muy buena y Mi primo siempre fue débil de carácter, respectivamente, observaremos lo siguiente: el primero de ellos posee una semántica idéntica a la oración de (28), mientras que el segundo de ellos suspende la predicación sobre el presente que sí se da en (29) y lo equipara igualmente a un Aoristo.

Cerraremos este apartado reflexionando sobre los siguientes enunciados, ofrecidos por García Fernández (2004: 41): Siempre \{estaba / estuvo\} enfermo. Indica el autor que el uso del pretérito imperfecto está justificado porque siempre expresa aquí habitualidad; esto es, las enfermedades eran frecuentes. En el caso del PI considera este mismo autor que se trata de una única enfermedad "durante todo el periodo". A la luz de los datos ofrecidos hasta ahora observamos que esta intuición puede plasmarse de un modo mucho más concreto: puesto que no consideramos que los estados puedan evolucionar en el tiempo (si se extendieran durante un periodo serían dinámicos), la interpretación temática de siempre que nosotros presentamos aquí permite establecer una conexión notable con la manera en la cual se conceptualiza la perfectividad de los estados. 


\section{Conclusión}

Este trabajo nos ha servido para reivindicar la postura ya avanzada por autores como Lope Blanch (1961) acerca de los diferentes niveles de gramaticalización del PPC en el ámbito hispánico. Básicamente se trata de que en el español estándar peninsular esta forma, que también expresa aspecto Perfecto, va camino de convertirse en la única opción para expresar el tiempo pretérito, en detrimento del PI $-\mathrm{y}$ de manera similar a un fenómeno que ya se ha consumado en el francés hablado-. Esto posee la siguiente implicación: en la variedad aludida, el PPC se ha convertido en la forma por defecto.

En las variedades latinoamericanas como el español de México, el PPC posee únicamente una semántica aspectual de Perfecto, pero en un significado iterativo que no se registra en el español europeo. Esta constatación revela una interesante línea de investigación: oraciones como Juan ya llegó dejan al descubierto un posible retroceso del PPC en contextos no iterados, dado que lo esperable es que los verbos télicos (y más aún en presencia del adverbio fasal correspondiente) remitieran a una interpretación de Perfecto. Nuestra hipótesis consiste, por tanto, en defender que verbos como comer nunca han aceptado en México la forma compuesta.

Partiendo de la base de que los predicados atélicos sólo pueden expresar el aspecto Aoristo, la presencia de adverbios como ya nos ha situado tras la pista de las funciones informativas: Yo ya he comido implica una información conocida que, por extensión, permite la formulación de oraciones como Yo ya he comido caviar. Esto nos lleva 
a considerar que el Experiencial posee originalmente una naturaleza temporal (y no aspectual); de manera que hemos planteado la posibilidad de que esta lectura no se dé en el ámbito latinoamericano.

\section{Bibliografía}

Alarcos Llorach, Emilio (1947), "Perfecto simple y compuesto en español", Revista de Filología Española, XXXI, pp. 108-139.

Bachelet Jeria, Michelle (2007), "Mensaje presidencial 21 de mayo 2007”. Disponible en línea: http:// www.bcn.cl/carpeta_temas_profundidad/temas_ profundidad.2008-03-27.7494909065/documentos/ MENSAJE\%20PRESIDENCIAL\%2021\%20DE\%20 MAYO\%20DE\%202007.pdf [consultada el 06 de diciembre 2014].

Berschin, Helmut (1976), Präteritum- und Perfektgebrauch im heutigen Spanisch, Tubinga, Max Niemeyer. Bertinetto, Pier M. (1986), Tempo, aspetto e azione nel verbo italiano, Florencia, Academia della Crusca. (2000), "The progressive in Romance, as compared with English", en Ö. Dahl (ed.), Tense and Aspect in the languages of Europe, Berlín / Nueva York, Mouton de Gruyter, pp. 559-604.

(2004), "Estativos, progresivos, habituales", en L. García Fernández y B. Camus Bergareche (eds.), El pretérito imperfecto, Madrid, Gredos, pp. 273-316. Aparecido originalmente en P. M. Bertinetto (1994), "Statives, 
progressives, and habituals: analogies and differences", Linguistics, 32, pp. 391-423.

Cartagena, Nelson (1999), “Los tiempos compuestos”, en I. Bosque y V. Demonte (dirs.), Gramática descriptiva de la lengua española, Madrid, Espasa Calpe, pp. 29352975.

Comrie, Bernard (1976), Aspect. An Introduction to the Study of Verbal Aspect and Related Problems, Cambridge, Cambridge University Press.

Detges, Ulrich (1999), "Wie entsteht Grammatik? Kognitive und pragmatische Determinanten der Grammatikalisierung von Tempusmarkern”, en J. Lang y I. Neumann-Holzschuh (eds.), Reanalyse und Grammatikalisierung in den romanischen Sprachen, Tubinga, Max Niemeyer, pp. 31-52. (2006), "Aspect and Pragmatics. The passé compose in Old French and the Old Spanish perfecto compuesto", en K. Eksell y T. Vinther (eds.), Change in verbal systems. Issues on explanation, Fráncfort del Meno, Peter Lang, pp. 47-72.

Dik, Simon C. (1987), "Copula auxiliarization: how and why", en M. Harris y P. Ramat (eds.), Historical development of auxiliaries, Berlín / Nueva York, Ámsterdam, Mouton de Gruyter, pp. 53-84.

(1997), The theory of functional grammar. Parte 1: The structure of the clause, Berlín / Nueva York, Mouton de Gruyter.

Fenn, Peter (1987), A semantic and pragmatic examination of the English Perfect, Tubinga, Gunter Narr. 
FLórez, Luis (1963), "El español hablado en Colombia y su atlas lingüístico", Thesaurus. Boletín del Instituto Caro $y$ Cuervo, 18/ 2, pp. 268-356.

García Fernández, Luis (2004), “El pretérito imperfecto: repaso histórico y bibliográfico”, en L. García Fernández y B. Camus Bergareche (eds.), El pretérito imperfecto, Madrid, Gredos, pp. 13-95.

(2006), “<Estar + gerundio>”, en L. García Fernández (dir.), Diccionario de perífrasis verbales, Madrid, Gredos, pp. 136-142.

GArrido, JoAquín (1992), "Expectations in Spanish and German adverbs of change", Folia Linguistica, 26, pp. 357-402.

Gómez Torrego, Leonardo (2003), Ejercicios de gramática normativa II, $3^{\text {a }}$ ed., Madrid, Arco / Libros.

Gutiérrez Araus, M. Luz (1995), Formas temporales del pasado en indicativo, Madrid, Arco / Libros.

HARris, MARTIN (1982), "The past simple and the present perfect in Romance", en N. Vincent y M. Harris (eds.), Studies in the Romance verb. Essays offered to Joe Cremona on the occasion of his $60^{\text {th }}$ birthday, Londres / Canberra, Croom Helm, pp. 42-70.

Haspelmath, Martin (1998), "Does grammaticalization need reanalysis?", Studies in Language, 22:2, pp. 315351.

(2004), "On the directionality in Language change with particular reference to grammaticalization", en $\mathrm{O}$. Fischer et al. (eds.), Up and down the cline-The nature the grammaticalization, Ámsterdam / Filadelfia, John Benjamins, pp. 17-44. 
Henderson, Carlos (2010), El Pretérito Perfecto Compuesto del español de Chile, Paraguay y Uruguay. Aspectos semánticos y discursivos, Stockholm University, tesis doctoral.

Hopper Paul J. y Elizabeth C. Traugott (2003), Grammaticalization, Cambridge, Cambridge University Press.

Instituto Cervantes (2013), Las 500 dudas más frecuentes del español, Madrid, Espasa Libros.

Kempas, Ilpo (2008a), "La elección de los tiempos verbales aorísticos en contextos hodiernales: sinopsis de datos empíricos recogidos en la España peninsular", en I. Olza et al. (eds.), Actas del XXXVII Simposio Internacional de la Sociedad Española de Lingüística, Pamplona, Servicio de Publicaciones de la Universidad de Navarra, pp. 397-408.

(2008b), "El pretérito perfecto compuesto y los contextos prehodiernales", en Á. Carrasco Gutiérrez (ed.), Tiempos compuestos y formas verbales complejas, Madrid / Fráncfort, Iberoamericana / Vervuert, pp. 231273.

Keniston, Hayward (1937), The syntax of Castilian prose. The sixteenth century, Chicago, University of Chicago. Klein, Wolfgang (1992), "The present perfect puzzle", Language, 68, pp. 225-252.

Laca, Brenda (2009), "Acerca de los perfectos en las variedades ibero-americanas", en F. Sánchez Miret (ed.), Romanística sin complejos. Homenaje a Carmen Pensado, Berna, Peter Lang, pp. 357-380.

LAmíQuiz IbáñeZ, VidAL (1998), "Lo lineal, lo blanco o negro y lo difuso (Acerca del método de la lingüística del 
Siglo xx)", Revista Española de Lingüística, 28, 1, pp. 29-47.

Lang, Jürgen e Ingrid Neumann-Holzschuh (1999), "Reanalyse und Grammatikalisierung. Zur Einführung in diesen Band", en J. Lang y I. Neumann-Holzschuh (eds.), Reanalyse und Grammatikalisierung in den romanischen Sprachen, Tubinga, Max Niemeyer pp. 1-17.

Lope Blanch, JuAn M. (1961), "Sobre el uso del pretérito en el español de México", Studia Philologica 2. Homenaje ofrecido a Dámaso Alonso por sus discípulos y amigos con ocasión de su 60 cumpleaños, Madrid, Gredos, pp. 373-385. Reimpreso bajo el mismo nombre en Juan M. Lope Blanch (2008), El español americano, México, El Colegio de México, pp. 17-32.

(1992), "Anomalía en la norma lingüística mexicana", en A. Vilanova (dir.), Actas del X Congreso de la Asociación Internacional de Hispanistas, Barcelona, PPU, pp. 1221-1226.

Martínez-Atienza, María (2008), "Dos formas de oposición en el ámbito románico entre el pretérito perfecto compuesto y el pretérito perfecto simple", en Á. Carrasco Gutiérrez (ed.), Tiempos compuestos y formas verbales complejas, Madrid / Fráncfort, Iberoamericana / Vervuert, pp. 203-229.

Moreno de Alba, José G. (2006), "Valores verbales de los tiempos pasados de indicativo y su evolución”, en C. Company Company (dir.), Sintaxis histórica de la lengua española. Primera parte: La frase verbal, México, Fondo de Cultura Económica / Universidad Nacional Autónoma de México, vol. 2, pp. 3-92. 
Moreno Burgos, Juan (2014), Estatividad y aspecto gramatical, Universität Regensburg, tesis doctoral.

Moreno Cabrera, Juan Carlos (2003), Semántica y gramática. Sucesos, papeles semánticos y relaciones sintácticas, Madrid, Antonio Machado libros.

Muller, Claude (1975), "Remarques syntactico-sémantiques sur certains adverbes de temps", Le Français Moderne, 43:1, pp. 12-38.

Pustejovsky, James (1991), "The syntax of event structure", en B. Levin y S. Pinker (eds.), Lexical and conceptual structure, Oxford, Blackwell, pp. 47-81.

Real Academia Española, Corpus de referencia del espanol actual [CREA], disponible en línea: http://www.rae. es [consutada el 24 de noviembre de 2014]. y Asociación de Academias de la Lengua EspaÑola (RAe y AAle) (2010), Nueva gramática de la lengua española. Manual, Madrid, Espasa Libros.

Reichenbach, Hans (1947), Elements of symbolic logic, Nueva York / Londres, The Free Press / Collier-Macmillan.

Rodríguez Louro, Celeste (2009), Perfect evolution and change: a sociolinguistic study of Preterit and Present Perfect usage in contemporary and earlier Argentina, University of Melbourne, tesis doctoral.

Rojo, Guillermo (1974), "La temporalidad verbal en español”, Verba, 1, pp. 68-149.

SÁnchez Prieto, Raúl (2011), "Futuro y pasado progresivo de indicativo en español y alemán", en C. Sinner et al. (eds.), Tiempo, espacio y relaciones espacio-temporales. 
Nuevas aportaciones de los estudios contrastivos, Fráncfort del Meno, Peter Lang, pp. 145-158.

Schwenter, Scott A. y Rena Torres Cacoullos (2008), "Defaults and indeterminacy in temporal grammaticalization: the 'perfect' road to perfective", Language Variation and Change, 20, pp. 1-39.

Seco, Manuel (2005), Diccionario de dudas y de dificultades de la lengua española, 10 a ed., Madrid, Espasa Calpe.

Soto, Guillermo y Carlos Zenteno (2010), "La relación en el discurso entre el pretérito perfecto compuesto y las expresiones evaluativas. Análisis de una cuenta pública presidencial”, Revista Signos, 43 (73), pp. 333-354. Squartini, Mario (1998), Verbal periphrases in Romance: aspect, actionality and grammaticalization, Berlín / Nueva York, Mouton de Gruyter.

Veiga, Alexandre (1992), "La no independencia funcional del aspecto en el sistema verbal español", Español Actual, 57, pp. 65-80.

Vendler, Zeno (1957), "Verbs and times", Philosophical Review, 56, pp. 143-160. Reproducido en Zeno Vendler (1967), Lingusitics in Philosophy, Ítaca / Nueva York, Cornwell University Press, pp. 97-121. 